\title{
Pelvic Floor Muscle Weakness
}

National Cancer Institute

\section{Source}

National Cancer Institute. Pelvic Floor Muscle Weakness. NCI Thesaurus. Code C78524.

A reduction in the strength of the muscles of the pelvic floor. 